\title{
Gravitational waves from brane-world inflation with induced gravity
}

\author{
Mariam Bouhmadi-López 6 Roy Maartens \\ Institute of Cosmology $\&$ Gravitation, University of Portsmouth, Portsmouth PO1 2EG, UK
}

(Dated: February 1, 2008)

\begin{abstract}
We calculate the amplitude of gravitational waves produced by inflation on a de Sitter brane embedded in five-dimensional anti-de Sitter bulk spacetime, extending previous calculations in RandallSundrum type cosmology to include the effect of induced gravity corrections on the brane. These corrections arise via a term in the brane action that is proportional to the brane Ricci scalar. We find that, as in the Randall-Sundrum case, there is a mass gap between the discrete zero-mode and a continuum of massive bulk modes, which are too heavy to be excited during inflation. We give the normalization of the zero-mode as a function of the Hubble rate on the brane and are thus able to calculate the high energy correction to the spectrum of gravitational wave (tensor) modes excited on large scales during inflation from initial vacuum fluctuations on small scales. We also calculate the amplitude of density (scalar) perturbations expected due to inflaton fluctuations on the brane, and show that the usual four-dimensional consistency relation for the tensor/scalar ratio remains valid for brane inflation with induced gravity corrections.
\end{abstract}

\section{INTRODUCTION}

String theory and $\mathrm{M}$ theory have motivated interest in brane-world models that describe extra-dimensional gravity. In these models, the observable four-dimensional (4D) universe is a brane hypersurface embedded in a higher-dimensional bulk space. A simple but rich phenomenology for cosmology is provided by the RandallSundrum (RS) models with a single brane in a fivedimensional $(5 \mathrm{D})$ anti de Sitter $\left(\mathrm{AdS}_{5}\right)$ spacetime. (For recent reviews, see Ref. [1].) Although the fifth dimension is infinite, the zero-mode of the $5 \mathrm{D}$ graviton, corresponding to $4 \mathrm{D}$ gravitational waves, is localized at low energies on the brane due to the warped geometry of the bulk. This allows one to recover general relativity in the low-energy limit.

Quantum corrections to the RS model are expected to arise from the induced coupling of brane matter to bulk gravitons. This is the so-called induced gravity (IG) effect, that leads to the appearance of terms proportional to the 4D Ricci scalar in the brane action [2, 3, 4]. In the Dvali, Gabadadze and Porrati (DGP) model [3] the induced gravity brane-world was put forward as an alternative to the RS one-brane model, in which general relativity was recovered, also despite an infinite extra dimension, but without warping in 5D Minkowski spacetime (the brane had no tension). In contrast to the RS case with high-energy modifications to general relativity, the DGP brane-world produced a low-energy modification, leading to late-time acceleration even in the absence of dark energy [5].

Here we will take a different viewpoint: we are mainly interested in the IG effect as a correction to the RS brane-

\footnotetext{
*Electronic address: mariam.bouhmadi@port.ac.uk

${ }^{\dagger}$ Electronic address: roy.maartens@port.ac.uk

${ }^{\ddagger}$ Electronic address: david.wands@port.ac.uk
}

world at high energies, rather than as a low-energy alternative (see also Refs. $6,6,7,8,9,10]$ ). In this case the IG effect will operate at high energies, above the brane tension, which is the threshold energy for RS modifications to general relativity. As a consequence there is no latetime IG modification to general relativity, but rather an early-time modification to the RS model. High-energy inflation will be subject to the IG effect, and we seek to determine in particular the consequent changes to the gravitational waves generated during inflation. If the IG correction becomes large so that it is dominant, then there is no high-energy RS regime in the early universe, and we recover the original DGP model.

During slow-roll inflation, zero-point quantum fluctuations of light fields (whose effective mass is less than the Hubble scale) are swept up to super-Hubble scales and acquire a nearly scale-invariant spectrum. In the RS brane-world, the zero-mode of the $5 \mathrm{D}$ graviton is a massless spin-2 field on the brane that represents $4 \mathrm{D}$ gravitational waves. But there are also massive modes on the brane that arise via the projection onto the brane of the $5 \mathrm{D}$ graviton. These massive modes could qualitatively alter the spectrum of gravitational waves generated during inflation. It turns out that there is a mass gap between the zero-mode and the the massive modes [1], so that the massive modes are too heavy to be excited and the spectrum is qualitatively the same as in general relativity. However, the amplitude of the zero-mode is boosted at high energies, compared with the standard 4D case [12].

We show that significant changes to the RS case are introduced by the IG term at high energies: the IG correction acts to limit the growth of amplitude, relative to the $4 \mathrm{D}$ case, as the energy scale of inflation grows. Then we investigate the scalar perturbations produced in inflation (assuming there is no contribution from 5D gravitons), and show that the scalar amplitude has the same qualitative behaviour as the tensor amplitude. We also show that the standard tensor-to-scalar consistency relation continues to hold in the presence of the IG cor- 
rection.

\section{FIELD EQUATIONS}

We assume that the 5D bulk contains only negative vacuum energy, and the gravitational field obeys Einstein gravity. The $4 \mathrm{D}$ brane has a positive brane tension, and an induced gravity term localized on it. Then the gravitational action is

$$
\begin{aligned}
\mathcal{S}= & \frac{1}{2 \kappa_{5}^{2}} \int d^{5} x \sqrt{-{ }^{(5)} g}\left[{ }^{(5)} R-2 \Lambda_{5}\right] \\
& +\int_{\text {brane }} d^{4} x \sqrt{-g}\left[-\sigma+\frac{\gamma}{2 \kappa_{4}^{2}} R+\mathcal{L}_{\text {matter }}\right],
\end{aligned}
$$

where $\gamma$ is a dimensionless constant controlling the strength of the IG correction, with $\gamma=0$ giving the RS model. The induced metric on the brane is $g_{a b}=$ ${ }^{(5)} g_{a b}-n_{a} n_{b}$, where $n^{a}$ is the unit normal, and $\sigma(\geq 0)$ is the brane tension, while $\Lambda_{5}(\leq 0)$ is the bulk cosmological constant. The fundamental energy scale of gravity is the $5 \mathrm{D}$ scale $M_{5}$, where $\kappa_{5}^{2}=8 \pi / M_{5}^{3}$. The $4 \mathrm{D}$ Planck scale $M_{4} \sim 10^{19} \mathrm{GeV}$ is an effective scale, fixed by the effective gravitational coupling constant $\kappa_{4}^{2}=8 \pi G=8 \pi / M_{4}^{2}$ on the brane at low energies (typically $M_{4} \gg M_{5}$ ). Note that for the low- and high-energy limits to yield a positive effective gravitational coupling constant, we require $0 \leq \gamma<1$.

The $5 \mathrm{D}$ field equations following from the bulk action are unchanged from the RS case:

$$
{ }^{(5)} G_{a b}=-\Lambda_{5}{ }^{(5)} g_{a b} .
$$

The IG correction term enters via the junction conditions at the brane. Assuming mirror $\left(\mathbb{Z}_{2}\right)$ symmetry about the brane, these are [7]

$$
K_{\mu \nu}-K g_{\mu \nu}=-\frac{\kappa_{5}^{2}}{2}\left(T_{\mu \nu}-\sigma g_{\mu \nu}-\frac{\gamma}{\kappa_{4}^{2}} G_{\mu \nu}\right),
$$

where $K_{\mu \nu}$ is the extrinsic curvature and $T_{\mu \nu}$ is the energy-momentum tensor of matter on the brane, which obeys the conservation equations [7]

$$
\nabla^{\nu} T_{\mu \nu}=0 .
$$

The length scale $\ell$ and energy scale $\mu$ associated with the bulk curvature are given by

$$
\Lambda_{5}=-\frac{6}{\ell^{2}}=-6 \mu^{2} .
$$

If we impose the RS fine-tuning for the brane tension, $\kappa_{5}^{2} \sigma=\sqrt{-6 \Lambda_{6}}$, this sets the brane cosmological constant to zero, and then we have [7, 10]

$$
\kappa_{4}^{2} \equiv \kappa_{5}^{2} \mu(1-\gamma) .
$$

The general form of the effective field equations projected on the brane is given in Ref. [7].
We will be interested in $4 \mathrm{D}$ tensor metric perturbations about a de Sitter brane (modelling extreme slow-roll inflation) in an $\mathrm{AdS}_{5}$ bulk. Such a brane is a solution of the junction equations (3) with a constant energy density on the brane, $\rho>0$, and hence a constant Hubble rate on the brane, $H$. The background space-time metric, i.e., an $\mathrm{AdS}_{5}$ bulk sliced into 4D slices with de Sitter geometry, may be written in the Gaussian normal form

$$
d s^{2}=n^{2}(y)\left[-d t^{2}+\exp \left(2 H_{\epsilon} t\right) d \vec{x}^{2}\right]+d y^{2} .
$$

The warp factor in the bulk is

$$
n(y)=\frac{H_{\epsilon}}{\mu} \sinh \left[\mu\left(y_{\epsilon}+\epsilon|y|\right)\right] .
$$

The brane is fixed at $y=0$ and we choose $n(0)=1$ on the brane, so that $y_{\epsilon}$ is given by

$$
y_{\epsilon}=\frac{1}{\mu} \operatorname{arcsinh} \frac{\mu}{H_{\epsilon}} .
$$

The Hubble parameter $H_{\epsilon}$ is given by the modified Friedman equation

$$
\begin{aligned}
& H_{\epsilon}^{2}=\frac{\kappa_{4}^{2}}{3 \gamma}\{\rho+\sigma \\
& \left.+\frac{6 \kappa_{4}^{2}}{\gamma \kappa_{5}^{4}}\left[1+\epsilon \sqrt{1+\frac{\gamma \kappa_{5}^{4}}{3 \kappa_{4}^{2}}\left(\rho+\sigma+\frac{\gamma \sigma^{2} \kappa_{5}^{4}}{12 \kappa_{4}^{2}}\right)}\right]\right\}
\end{aligned}
$$

where we have imposed Eq. (6). In the expressions (7)(10), $\epsilon= \pm 1$ corresponds to the choice of sign for the square-root in Eq. (10). We will refer to $\epsilon=+1$ as the positive branch and $\epsilon=-1$ as the negative branch. In the following we take the energy density, $\rho$, to be constant, corresponding to the inflaton energy density in the extreme slow roll limit. We recover the Randall-Sundrum case [12 in the limit $\gamma \rightarrow 0$, but only on the negative branch.

On the positive branch, the bulk coordinate $y$ can take any real value and the warp factor $n(y)$ reaches its minimum value at the brane. On the other hand, in the negative branch, the bulk coordinate is bounded such that $|y| \leq y_{-}$, where $|y|=y_{-}$corresponds to the location of the Cauchy horizon, where $n\left(y_{-}\right)=0$. In this case, the warp factor has its maximum at the brane. We recover the original RS model, with a Minkowski brane, as $H \rightarrow 0$ and $y_{-} \rightarrow \infty$ for $\rho \rightarrow 0$, on the negative branch. The original DGP model with $H_{+}>0$ is obtained in the low energy limit on the positive branch as $\rho+\sigma \rightarrow 0$.

For later convenience, we introduce the conformal bulk coordinate, with $d z=d y / n(y)$,

$$
z(y)=-\frac{\epsilon}{\mu} \operatorname{sgn}(y) \sinh \mu y_{\epsilon} \ln \operatorname{coth}\left[\frac{\mu}{2}\left(y_{\epsilon}+\epsilon|y|\right)\right] .
$$

For the positive branch $0<|z| \leq z_{b+}$, where $z_{b+}$ corresponds to the location of the brane, $z_{b+}=|z(0)|$. When $|z| \rightarrow 0$, the bulk coordinate $y$ diverges. For the negative branch, the conformal bulk coordinate is unbounded, 
$|z| \geq z_{b-}$, where $z_{b-}$ gives the location of the brane. In this case, the Cauchy horizons are located at $|z| \rightarrow+\infty$. Finally, the warp factor becomes

$$
n(z)=\frac{H_{\epsilon}}{\mu \sinh H_{\epsilon}|z|} .
$$

\section{BULK METRIC PERTURBATIONS}

We now consider tensor metric perturbations (from the viewpoint of a 4 D observer $),{ }^{(5)} g_{a b} \rightarrow{ }^{(5)} g_{a b}+\delta{ }^{(5)} g_{a b}$. The perturbed metric is 12

$$
d s^{2}=n^{2}(y)\left[-d t^{2}+e^{-2 H_{\epsilon} t}\left(\delta_{i j}+h_{i j}\right) d x^{i} d x^{j}\right]+d y^{2},
$$

where $h_{i j}$ is transverse and traceless. The wave equation in the bulk for the perturbations is

$$
\delta^{(5)} G_{b}^{a}=0
$$

the same as in the RS case. This means that the bulk mode solutions for metric perturbations will be the same as in the RS case [12], but the IG junction conditions will introduce changes to the normalization and amplitudes of the modes, as discussed below.

We decompose the general metric perturbation into Fourier modes

$$
h_{i j}(t, \vec{x}, y)=E(t, y) e_{i j}(\vec{x})
$$

where $e_{i j}$ is a transverse traceless harmonic on the spatially flat 3 -space, i.e., $\vec{\nabla}^{2} e_{i j}=-k^{2} e_{i j}$. Then the equation of motion for $E$ is 12 ]

$$
\ddot{E}+3 H_{\epsilon} \dot{E}+k^{2} e^{-2 H_{\epsilon} t} E-n^{2}\left(E^{\prime \prime}+4 \frac{n^{\prime}}{n} E^{\prime}\right)=0,
$$

where a dot denotes a derivative with respect to $t$ and a prime a derivative with respect to $y$. The perturbation $E$ may be decomposed into Kaluza-Klein (KK) modes

$$
E(t, y)=\int d m \psi_{m}(t) \mathcal{E}_{m}(y)
$$

and the wave equation (16) then separates to give

$$
\begin{aligned}
\ddot{\psi}_{m}+3 H_{\epsilon} \dot{\psi}_{m}+\left[k^{2} e^{-2 H_{\epsilon} t}+m^{2}\right] \psi_{m} & =0 \\
\left(n^{4} \mathcal{E}_{m}^{\prime}\right)^{\prime}+m^{2} n^{2} \mathcal{E}_{m} & =0 .
\end{aligned}
$$

Although the massive modes of the tensor perturbations satisfy the same bulk wave equation as in the RS case 12 , the junction condition at the brane is very different. The tensor part of Eq. (3) implies

$$
\delta K^{\mu}{ }_{\nu}=\frac{\gamma \kappa_{5}^{2}}{2 \kappa_{4}^{2}} \delta G^{\mu}{ }_{\nu}=\frac{\gamma}{2 \mu(1-\gamma)} \delta G^{\mu}{ }_{\nu}
$$

where we have neglected any tensor contributions of the anisotropic stress exerted by matter on the brane, and the second equality follows from Eq. (6). In the $\mathrm{RS}$ case $(\gamma \rightarrow$ $0)$, we recover the Neumann boundary condition. On the brane, the non-vanishing components of the Einstein tensor are given by [12, 13]

$$
\delta G^{i}{ }_{j}=\frac{1}{2}\left[\dddot{h}^{i}{ }_{j}+3 H_{\epsilon} \dot{h}^{i}{ }_{j}-e^{-2 H_{\epsilon} t} \vec{\nabla}^{2} h^{i}{ }_{j}\right],
$$

and consequently, the boundary condition for $\mathcal{E}_{m}$ is

$$
\mathcal{E}_{m}^{\prime}(0)=-\frac{m^{2} \gamma}{2 \mu(1-\gamma)} \mathcal{E}_{m}(0)
$$

It is important to note that this boundary condition depends on the mass of the modes, $\mathrm{m}^{2}$, due to the IG term. Only the zero-mode, $m=0$, has the same boundary condition as in the RS case. As a result of this new feature, the scalar product of the eigenmode functional space has to include suitable boundary terms. This is qualitatively the same as the case of Gauss-Bonnet modifications to the RS model [14, 15].

In what follows we show that the eigenmodes resulting from Eqs. (19) and (22) are orthonormal with respect to the following scalar product:

$$
\begin{aligned}
\left(\mathcal{E}_{m}, \mathcal{E}_{\tilde{m}}\right) & =2 \int_{0}^{U_{\epsilon}} d y n^{2} \mathcal{E}_{m} \mathcal{E}_{\tilde{m}}+\frac{\gamma}{\mu(1-\gamma)} \mathcal{E}_{m}(0) \mathcal{E}_{\tilde{m}}(0) \\
& =\delta(m, \tilde{m})
\end{aligned}
$$

where $U_{+}=\infty, U_{-}=y_{-}$, and $\delta(m, \tilde{m})$ denotes a Kronecker symbol for the discrete modes and a Dirac distribution for the continuous ones. For $\gamma \rightarrow 0$ the scalar product reduces to the one used in the RS case.

\section{KALUZA-KLEIN MODES}

We consider a single de Sitter brane embedded in AdS space so that we only impose one boundary condition, Eq. (22). One could also consider the presence of a second brane [16, 17], with a corresponding second boundary condition, which would yield a discrete spectrum of bulk modes.

Our discussion of the spectrum of bulk modes will follow closely that of Ref. [15]. Defining $\phi_{m} \equiv n^{3 / 2} \mathcal{E}_{m}$, we rewrite the wave equation (19) in a way that incorporates the junction condition (22):

$$
-\mathcal{D}_{(+)}\left[q(z) \mathcal{D}_{(-)} \phi_{m}(z)\right]=m^{2} w(z) \phi_{m}(z),
$$

where

$$
\mathcal{D}_{( \pm)}=\frac{d}{d z} \pm \frac{3}{2} \frac{1}{n} \frac{d n}{d z}
$$

and

$$
\begin{aligned}
q & =\theta\left[\epsilon\left(z_{b \epsilon}-z\right)\right]-\epsilon \theta\left[-z-z_{b \epsilon}\right] \\
w & =q(z)+\frac{\gamma}{\mu(1-\gamma)} \delta\left(z+\epsilon z_{b \epsilon}\right) .
\end{aligned}
$$


Here $\theta$ is the heaviside function, and the \pm subscripts in $\mathcal{D}_{ \pm}$are not to be confused with the branch parameter $\epsilon$.

From Eqs. (12), (26) and (27) it follows that for $|z| \neq$ $z_{b \epsilon}$, we have $q=w=1$. Thus for $|z| \neq z_{b \epsilon}$, Eq. [24] reduces to the Schrödinger-type equation,

$$
\begin{aligned}
-\mathcal{D}_{(+)} \mathcal{D}_{(-)} \phi_{m} & =-\frac{d^{2} \phi_{m}}{d z^{2}}+\left[\frac{15}{4} \frac{H_{\epsilon}^{2}}{\sinh ^{2} H_{\epsilon} z}+\frac{9}{4} H_{\epsilon}^{2}\right] \phi_{m} \\
& =m^{2} \phi_{m},
\end{aligned}
$$

exactly as in the RS case $(\gamma \rightarrow 0)$ 11, 12]. is

The boundary condition, Eq. (22), for $\phi_{m}$ at the brane

$$
\mathcal{D}_{(-)} \phi_{m}\left(-\epsilon z_{b \epsilon}\right)=-m^{2} \frac{\gamma}{2 \mu(1-\gamma)} \phi_{m}\left(z_{b \epsilon}\right)
$$

(Note that in the negative branch, $y \rightarrow 0^{+}$implies $z \rightarrow$ $z_{b-}^{+}$, while for the positive branch, $y \rightarrow 0^{+}$implies $z \rightarrow$ $-z_{b+}^{+}$.) Equation (29) may also be obtained by matching the distributional parts of Eq. (24). It may be checked that the eigenmodes resulting from Eqs. (28) and (29) are orthonormal with respect to the scalar product Eq. (23), which results from

$$
\left(\phi_{m}, \phi_{\tilde{m}}\right)=\int_{\mathrm{bulk}} d z w \phi_{m} \phi_{\tilde{m}}
$$

when $\mathbb{Z}_{2}$-symmetry is imposed and the distributional term in Eq. (27) is taken into account.

We now investigate the spectrum of modes resulting from Eqs. (28) and (29). There is the same zero-mode solution as in the RS case 12 ]

$$
\phi_{0}(z)=C_{\epsilon} n^{3 / 2}(z)
$$

where $\mathcal{E}_{0}=C_{\epsilon}$ is a constant. This zero-mode solution obeys the boundary condition (29) for both the positive and negative branches in Eq. (10).

The zero mode in the negative branch is normalizable as in the RS case. Using Eqs. (30) and (31), the condition $\left(\phi_{0}, \phi_{0}\right)=1$ on the negative branch gives the normalization constant $C=C\left(H_{-} / \mu\right)$ as a function of the Hubble rate relative to the $\mathrm{AdS}$ scale, $\mu$, where

$$
C_{-}^{-2}(x)=\frac{\gamma}{\mu(1-\gamma)}+\frac{1}{\mu}\left[\sqrt{1+x^{2}}-x^{2} \operatorname{arcsinh} \frac{1}{x}\right]
$$

This reduces to the RS result [12] when $\gamma=0$.

However, the zero-mode is not normalizable in the positive branch (for a single brane), since $\phi_{0}$ diverges as $z \rightarrow 0$. We will henceforth restrict the analysis to the negative branch, and drop the negative subscript, i.e., $H_{-} \equiv H, z_{b-} \equiv z_{b}$.

As in the RS case [11, 12], the potential in the Schrödinger equation (28) approaches $\frac{9}{4} H^{2}$, as $|z| \rightarrow \infty$, which gives the threshold between light modes, with $m^{2}<\frac{9}{4} H^{2}$, and heavy modes, with $m^{2}>\frac{9}{4} H^{2}$. For heavy modes, the two linearly independent solutions of
Eq. (28) oscillate with constant amplitude as $|z| \rightarrow \infty$. The boundary condition Eq. (29) gives $\phi_{m}(z)$ as a particular combination of these two solutions, for every $m$. Thus we have a continuous spectrum of normalizable modes with $m^{2}>\frac{9}{4} H^{2}$.

For light modes, the general solution of the Schrödinger equation (28) contains an asymptotically growing exponential and a decaying exponential as $|z| \rightarrow \infty$. Thus we would expect only a discrete spectrum of normalizable modes corresponding to particular values of $m^{2} \leq \frac{9}{4} H^{2}$ for which the junction condition (29) selects only the asymptotic decaying mode. This is exactly what happens to give the normalizable zero-mode (31) for the negative branch in Eq. (10).

In order to see whether the junction conditions allow for normalizable light modes other than the zero-mode, we introduce new mode functions, following Ref. 15],

$$
\Phi_{m}(z)=\mathcal{D}_{(-)} \phi_{m}(z) .
$$

These are the partners of the modes $\phi_{m}$ in supersymmetric quantum mechanics [18], and have the same spectrum except for the zero-mode, $\Phi_{0}$, which vanishes identically, by Eqs. (25) and (31). Acting on Eq. (28) with the operator $\mathcal{D}_{(-)}$, we obtain the wave equation for $\Phi_{m}$

$$
\begin{aligned}
-\mathcal{D}_{(-)} \mathcal{D}_{(+)} \Phi_{m} & =-\frac{d^{2} \Phi_{m}}{d z^{2}}+\left[\frac{3}{4} \frac{H^{2}}{\sinh ^{2} H z}+\frac{9}{4} H^{2}\right] \Phi_{m} \\
& =m^{2} \Phi_{m} .
\end{aligned}
$$

The junction condition for $\Phi_{m}$ follows from Eqs. (28) and (29),

$$
\frac{d \Phi_{m}}{d z}\left(z_{b}\right)=\left[\frac{3}{2} \sqrt{H^{2}+\mu^{2}}+\frac{2 \mu(1-\gamma)}{\gamma}\right] \Phi_{m}\left(z_{b}\right),
$$

for $\gamma \neq 0$, while Eqs. (29) and (33) show that $\Phi_{m}\left(z_{b}\right)=0$ for $\gamma=0$. The important simplification here is that the boundary condition no longer involves the mass of the modes, and reduces to Dirichlet-type for $\gamma=0$. Multiplying Eq. (34) by $\Phi_{m}$ and integrating by parts, we find that

$$
\begin{gathered}
\left(m^{2}-\frac{9}{4} H^{2}\right) \int_{z_{b}}^{\infty} d z \Phi_{m}^{2}=\frac{3}{4} H^{2} \int_{z_{b}}^{\infty} d z \frac{\Phi_{m}^{2}}{\sinh ^{2} H z} \\
+\int_{z_{b}}^{\infty} d z \Phi_{m}^{\prime 2}+\left[-\Phi_{m} \Phi_{m}^{\prime}\right]_{z_{b}}^{\infty}
\end{gathered}
$$

Any normalizable light mode, $\phi_{m}$, with $m^{2}<\frac{9}{4} H^{2}$, must decrease exponentially as $|z| \rightarrow \infty$, and so must its partner, $\Phi_{m}$. The corresponding upper boundary term at infinity in Eq. (36) therefore vanishes. The lower boundary term on the brane, according to Eq. (35), is positive, remembering that we have restricted our analysis to $0 \leq \gamma<1$. In this case, the right-hand side of Eq. (36) is positive, while the left-hand side is negative, except for $\Phi_{m}=0$, i.e., for $m=0$. We can therefore conclude that for $0 \leq \gamma<1$, there are no normalizable light modes in 
the mass gap. The spectrum of KK modes in the negative branch of Eq. (10) is the same as the RS case, i.e., it consists of the massless bound-state zero-mode, and a continuum of states with $m>\frac{3}{2} H$.

As in the RS case $(\gamma \rightarrow 0)[11,12$ and as in the case of Gauss-Bonnet gravity in the bulk [15], we have only one discrete light mode with $m^{2}=0$ and a then mass-gap before the continuum of heavy modes. On the other hand, we have shown that there is no zero-mode for the positive branch, $\epsilon=+1$, and it remains to be seen whether or not there are light modes with $m^{2}<\frac{9}{4} H^{2}$ in this case.

\section{QUANTUM FLUCTUATIONS ON THE BRANE}

Having identified the normalizable bulk modes for a single de Sitter brane in an anti-de Sitter bulk, we will now estimate the spectrum of graviton fluctuations generated in de Sitter inflation on the brane for the negative branch in Eq. (10). We treat each normalizable mode as a quantum field in four dimensions, as in the RS case [12, 19]. Taking these $4 \mathrm{D}$ fields to be in an initial vacuum state on small scales is consistent with taking an incoming AdS vacuum state in the five-dimensional viewpoint [20].

Massive modes with $m^{2}>\frac{9}{4} H^{2}$ remain underdamped even on large scales, and fluctuations are strongly suppressed on super-horizon scales. They remain in their vacuum state [12, 19].

However initial vacuum fluctuations in the zero-mode become over-damped as they are stretched beyond the $\mathrm{F}_{\gamma}^{2}$ Hubble scale. The zero-mode thus acquires a spectrum of classical perturbations on super-horizon scales. For $m^{2}=0$, the effective action has the standard form of $4 \mathrm{D}$ general relativity, except for the overall factor $\kappa_{5}^{2}$ instead of $\kappa_{4}^{2}$ [15, 17], which rescales the amplitude of quantum fluctuations accordingly 12, 15]. The amplitude of the zero-mode metric fluctuations on the brane, $\phi_{0}\left(z_{b}\right)=C(H / \mu)$, where $C(H / \mu)$ is given in Eq. (32), then introduces a further rescaling relative to the $4 \mathrm{D}$ result, which is dependent on the Hubble scale relative to the AdS scale. Following the notation of Ref. [21], the amplitude of gravitational waves produced on superhorizon scales on the brane is thus given by

$$
A_{T}^{2}=\frac{2 \kappa_{4}^{2}}{25}\left(\frac{H}{2 \pi}\right)^{2} F_{\gamma}^{2}(H / \mu)
$$

where the correction to standard $4 \mathrm{D}$ general relativity is given by

$$
F_{\gamma}^{2}(H / \mu)=\frac{\kappa_{5}^{2}}{\kappa_{4}^{2}} C^{2}(H / \mu)
$$

From Eq. (32), we have

$$
F_{\gamma}^{-2}(x)=\gamma+(1-\gamma)\left[\sqrt{1+x^{2}}-x^{2} \operatorname{arcsinh} \frac{1}{x}\right] \text {. }
$$

This correction depends on the IG coupling (through the parameter $\gamma$ ) and on the energy scale at which inflation occurs, relative to the $5 \mathrm{D}$ curvature scale $\mu$. It reduces to the result of Ref. [12] for the RS case $(\gamma \rightarrow 0)$.

When $x \equiv H / \mu \rightarrow 0$, we have $F_{\gamma} \rightarrow 1$ and we recover the standard $4 \mathrm{D}$ result [21]. The amplitude of the normalized zero-mode on the brane gives the ratio between the effective 4D Newton constant at low energies, $\kappa_{4}^{2}$, and the $5 \mathrm{D}$ constant, $\kappa_{5}^{2}$.

For $0<\gamma<1$ we find that tensor fluctuations are enhanced relative to the $4 \mathrm{D}$ result $\left(F_{\gamma}>1\right)$ at high energies. However, unlike the RS case, the amplitude of the tensor zero-mode relative to the $4 \mathrm{D}$ general relativity result is bounded, $1 \leq F_{\gamma}^{2}<1 / \gamma$. For $H \gg \mu$ and $0<\gamma<1$, we have

$$
F_{\gamma}^{2}(H / \mu) \approx \frac{1}{\gamma}
$$

while the RS case $(\gamma=0)$ yields 12

$$
F_{0}^{2}(H / \mu) \approx \frac{3}{2} \frac{H}{\mu} .
$$

The qualitative behaviour is illustrated in Fig. 1 for $\gamma=$ 0.1 .

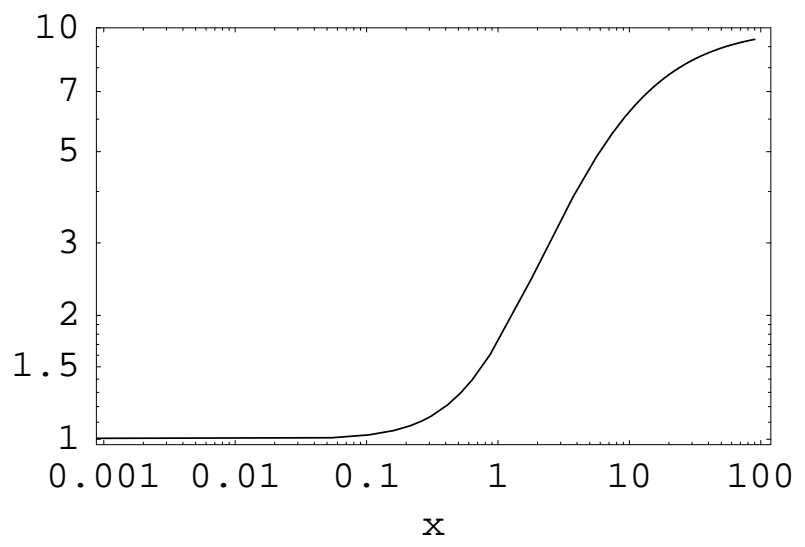

FIG. 1: The dimensionless amplitude $F_{\gamma}^{2}$ of the tensor zeromode relative to the $4 \mathrm{D}$ general relativity result, plotted against the dimensionless energy scale of inflation, $H / \mu$, for $\gamma=0.1$.

\section{THE TENSOR TO SCALAR RATIO}

The contribution of tensor perturbations to cosmic microwave background (CMB) anisotropies is an important quantity for constraining inflationary models [22]. In the RS case, both tensor and scalar perturbations on the brane are enhanced relative to a $4 \mathrm{D}$ inflation model at a fixed energy scale, but the tensors are enhanced less and thus the relative tensor contribution is suppressed in comparison with the $4 \mathrm{D}$ case [23]. In this section we 
compute the scalar perturbation amplitude $A_{S}$ including the effect of the IG term..

In standard, slow-roll inflationary models driven by a single inflaton field there is a consistency relation between the contribution to the CMB from scalar (density) perturbations and tensor modes (gravitational waves). (For a review, see, e.g., Ref. 21]). To lowest order in the slow-roll approximation, the ratio of the tensor to scalar perturbations is given by

$$
\frac{A_{T}^{2}}{A_{S}^{2}}=-\frac{1}{2} n_{T},
$$

where $n_{T} \equiv d \ln A_{T}^{2} / d \ln k$ represents the tilt of the tensorial spectrum and $k$ is comoving wavenumber.

An identical relation holds in 4D scalar-tensor and other generalized Einstein theories 24], and also in the RS scenario [25, 26], and in a 5D brane-world model where the radion field is stabilized [27]. Formally, the degeneracy in the brane-world models arises because the function that parametrizes the corrections to the gravitational wave amplitude satisfies a particular first-order differential equation [25, 28].

Given the potential importance of the consistency relation as a way of reducing the number of independent inflationary parameters, and of testing the inflationary scenario, it is important to investigate whether the above degeneracy is lifted when IG effects are included in the bulk action as a correction to the RS model.

\section{A. Scalar perturbations from IG brane inflation}

We will follow the approach used in Ref. [23] to calculate amplitude of scalar (density) perturbations due to slow-roll inflation on the brane in RS gravity. This has recently been applied to study the amplitude of scalar perturbations produced during inflation on the brane for Gauss-Bonnet [15] and induced gravity corrections [8, 9].

To estimate the primordial density perturbation produced by inflaton field fluctuations on the brane, to lowest order in a slow-roll approximation, it is sufficient to use the amplitude of quantum fluctuations of an effectively massless scalar field in an unperturbed de Sitter spacetime. This gives the classic result $\left\langle\delta \phi^{2}\right\rangle=$ $(H / 2 \pi)^{2}$ for modes exiting the Hubble scale, with comoving wavenumber $k=a H$, and entering the overdamped long-wavelength regime. The field fluctuation then determines the spatial curvature perturbation on uniformenergy density hypersurfaces, $\zeta=-H \delta \phi / \dot{\phi}$. Here and in similar expressions in this section, equality is to be understood as equality at the lowest order in the slow-roll approximation.

The curvature perturbation, $\zeta$, then remains constant for adiabatic perturbations on large scales. This holds as a direct consequence of the local conservation of energymomentum on the brane [29, 30] and, in particular, is independent of gravitational field equations. Hence we can calculate $\zeta$ at Hubble-exit during inflation and relate it directly to the primordial density perturbation on large scales, as for instance observed in the CMB anisotropies, so long as the perturbations are strictly adiabatic. This is necessarily the case for slow-roll, single field inflation on the brane so long as there are no additional light scalar degrees of freedom such as might arise from bulk metric perturbations in a brane-world scenario. This in turn is true in the extreme slow-roll limit in single brane Randall-Sundrum models and we expect it to remain true when induced gravity corrections are included. There is no scalar zero-mode and we have seen for the tensor perturbations that all the normalizable massive modes have $m^{2}>\frac{9}{4} H^{2}$ and remain suppressed during inflation.

Consequently, the amplitude of a given mode that reenters the Hubble radius long after inflation is given by [21]

$$
A_{S}^{2}=\frac{4}{25}\left\langle\zeta^{2}\right\rangle=\frac{H^{4}}{25 \pi^{2} \dot{\varphi}^{2}} .
$$

During slow-roll inflation the scalar field equation of motion, $\dot{\varphi}=-V^{\prime}(\varphi) / 3 H$, implies that the amplitude of scalar perturbations can be written as

$$
A_{S}^{2}=\frac{9}{25 \pi^{2}} \frac{H^{6}}{V^{\prime 2}} .
$$

Using Eqs. (10) and (6), we write this as

$$
A_{S}^{2}=\frac{\kappa_{4}^{6} V^{3}}{75 \pi^{2} V^{\prime 2}} G_{\gamma}^{2}(H / \mu),
$$

where the correction to the standard $4 \mathrm{D}$ result is given by

$$
G_{\gamma}^{2}(x)=\frac{x^{6}}{\left\{\gamma x^{2}-2(1-\gamma)\left[1-\sqrt{1+x^{2}}\right]\right\}^{3}} .
$$

The behaviour of $G_{\gamma}^{2}(x)$ is illustrated in Fig. 2. At low energies, $H / \mu \rightarrow 0$, we have $G_{\gamma}^{2}(H / \mu) \rightarrow 1$ and we recover the standard $4 \mathrm{D}$ result. At higher energies we begin to see an enhancement as in the RS case, but the enhancement of perturbations is bounded when we include the IG correction, just as we saw in the case of tensor perturbations. For $H \gg \mu$ and $0<\gamma<1$ we obtain

$$
G_{\gamma}^{2}(H / \mu) \approx \frac{1}{\gamma^{3}} .
$$

Equations (40) and (47) show that the scalars are more strongly enhanced at high energies than the tensors, so that the tensor/scalar ratio is suppressed at high energies relative to the standard $4 \mathrm{D}$ result.

\section{B. Consistency relation}

To see whether the consistency relation (42) is broken by induced gravity, we need to calculate the scaledependence of the tensor spectrum,

$$
n_{T}=\frac{d \ln A_{T}^{2}}{d \ln k} .
$$




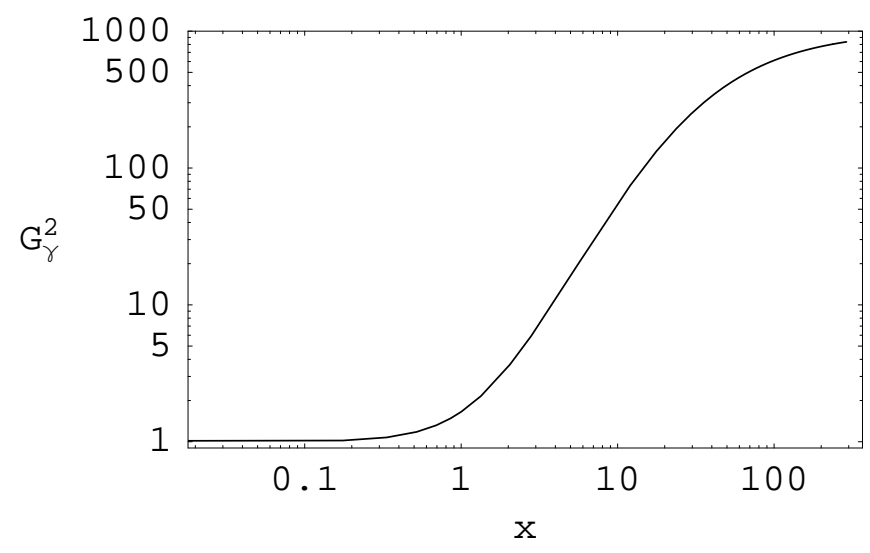

FIG. 2: The dimensionless amplitude $G_{\gamma}^{2}$ of density perturbations relative to the $4 \mathrm{D}$ general relativity result, plotted against the dimensionless energy scale of inflation, $H / \mu$, for $\gamma=0.1$.

We thus differentiate Eq. (37) with respect to comoving wavenumber $k(\varphi)=a(\varphi) H(\varphi)$. In the extreme slow-roll limit, variations in the Hubble parameter $H$ are negligible relative to changes in the scale factor and hence the spectral index can be written as

$$
n_{T}=-\frac{d \ln \left(H F_{\gamma}\right)^{-2}}{d \ln H} \frac{d \ln H}{d \ln a} .
$$

It is convenient to rewrite the scalar amplitude (44) in the form

$$
A_{S}^{2}=-\frac{3}{25 \pi} H^{3} \frac{d H}{d V}\left(\frac{d \ln H}{d \ln a}\right)^{-1}
$$

where we have used the scalar field slow-roll equation $3 H \dot{\varphi}=-V^{\prime}$, rewritten in the unusual form [15]

$$
V^{\prime 2}=-3 H^{3} \frac{d V}{d H} \frac{d \ln H}{d \ln a} .
$$

Then Eqs. (37), (49) and (50) can be combined to give

$$
\frac{A_{T}^{2}}{A_{S}^{2}}=-\frac{Q}{2} n_{T}
$$

where

$$
Q=-\frac{\kappa_{4}^{2}}{3 H} \frac{d V}{d H} F_{\gamma}^{2}\left[\frac{d \ln \left(H F_{\gamma}\right)^{-2}}{d \ln H}\right]^{-1} .
$$

For the consistency relation (42) to hold, we require $Q=1$. This amounts to a first-order differential relation between $H(V)$ and $F_{\gamma}(H / \mu)$ :

$$
\frac{d}{d V}\left(3 H^{2}\right)=\kappa_{4}^{2} F_{\gamma}^{2}\left(1+\frac{d \ln F_{\gamma}}{d \ln H}\right)^{-1} .
$$

It is easy to see that this is satisfied at low energies, where $F_{\gamma} \rightarrow 1$ and $3 H^{2} \rightarrow \kappa_{4}^{2} V$, and we recover the standard
$4 \mathrm{D}$ case. It is far from obvious that this continues to hold at higher energies. In fact one can show that the IG brane-world correction to the tensor power spectrum, Eq. (39), satisfies a first-order differential equation:

$$
\frac{d}{d \ln x}\left[\ln \left(x F_{\gamma}\right)^{-2}\right]=-2 F_{\gamma}^{2}\left[\gamma+\frac{1-\gamma}{\sqrt{1+x^{2}}}\right] .
$$

Combining the modified Friedmann equation (10) and Eq. (55), it can then be shown that Eq. (53) implies $Q=1$. Consequently, the brane gravity model with IG correction (in the negative branch) does indeed satisfy the consistency relation (42) for any value of $H / \mu$. This extends the result originally found in Ref. 25, 26] for the RS where $\gamma=0$, to any IG correction term with $0 \leq \gamma<1$.

\section{CONCLUSIONS}

Brane-world inflation models allows one to explore some of the cosmological implications of ideas arising from string and $M$ theory. The extra-dimensional nature of gravity introduces new features that need to be computed and then subjected to the constraints from high-precision cosmological data. We have determined the corrections to the standard results for tensor perturbations that are generated during slow-roll inflation at energies where brane effects become dominant. This has previously been done for the Randall-Sundrum braneworld. We have introduced an induced gravity term, as a correction to the gravitational action. This correction leads to significant qualitative changes.

The 5D wave equation and its fundamental solutions are not changed by the IG term. The spectrum contains a normalizable zero-mode and a continuous tower of massive modes after a mass gap, $m>\frac{3}{2} H$, as in the RS case. The massive modes are not excited during inflation, as in the RS case. However, the IG term changes the boundary conditions at the brane, and therefore changes the normalization of the zero-mode, as shown by Eq. (32). This has the consequence of enhancing the amplitude of tensor perturbations (gravitational waves) produced by de Sitter inflation on the brane, Eq.(37), relative to the standard 4D result. Unlike the RS case, the relative enhancement at high energies is bounded in the presence of a IG correction term. However, as in the RS case, we find that the standard consistency relation between the tensor-scalar ratio and the tilt of the tensor spectrum remains unchanged.

\section{Acknowledgements:}

We thank Lefteris Papantonopoulos for helpful discussions. MBL is funded by the Spanish Ministry of Education, Culture and Sport (MECD), and partly supported by DGICYT under Research Project BMF2002 03758. 
RM is supported by PPARC and DW is supported by the Royal Society.

[1] D. Langlois, arXiv:astro-ph/0301022 P. Brax and C. van de Bruck, Class. Quantum Grav. 20, R201 (2003) arXiv:hep-th/0303095; R. Maartens, Liv. Rev. Rel. 7 (2004) arXiv:gr-qc/0312059 ; P. Brax, C. van de Bruck, and A-C. Davis, arXiv:hep-th/0404011

[2] H. Collins and B. Holdom, Phys. Rev. D 62, 105009 (2000) arXiv:hep-ph/0003173.

[3] G. R. Dvali, G. Gabadadze, and M. Porrati, Phys. Lett. B485, 208 (2000) arXiv:hep-th/0005016.

[4] Y. Shtanov, arXiv:hep-th/0005193 S. Nojiri and S. D. Odintsov, JHEP 0007, 049 (2000) arXiv:hep-th/0006232; N. J. Kim, H. W. Lee and Y. S. Myung, Phys. Lett. B 504, 323 (2001) arXiv:hep-th/0101091; G. Kofinas, JHEP 0108, 034 (2001) arXiv:hep-th/0108013.

[5] C. Deffayet, Phys. Lett. B502, 199 (2001) arXiv:hep-th/0010186; C. Deffayet, G. R. Dvali and G. Gabadadze, Phys. Rev. D 65, 044023 (2002) arXiv:astro-ph/0105068; C. Deffayet, S. J. Landau, J. Raux, M. Zaldarriaga and P. Astier, Phys. Rev. D 66, 024019 (2002) arXiv:astro-ph/0201164; V. Sahni and Y. Shtanov, Int. J. Mod. Phys. D 11, 1515 (2000) arXiv:gr-qc/0205111; U. Alam and V. Sahni, arXiv:astro-ph/0209443 R. G. Vishwakarma and P. Singh, Class. Quantum Grav. 20, 2033 (2003) arXiv:astro-ph/0211285.

[6] E. Kiritsis, N. Tetradis and T. N. Tomaras, JHEP 0203, 019 (2002) arXiv:hep-th/0202037.

[7] K. Maeda, S. Mizuno, and T. Torii, Phys. Rev. D 68, 024033 (2003) arXiv:gr-qc/0303039.

[8] E. Papantonopoulos and V. Zamarias, arXiv:gr-qc/0403090

[9] H. s. Zhang and R. G. Cai, arXiv:hep-th/0403234

[10] M. Bouhmadi-López and D. Wands, in preparation.

[11] J. Garriga and M. Sasaki, Phys. Rev. D62, 043523 (2000) arXiv:hep-th/9912118.

[12] D. Langlois, R. Maartens, and D. Wands, Phys. Lett. B489, 259 (2000) arXiv:hep-th/0006007.

[13] H. A. Bridgman, K. A. Malik and D. Wands, Phys. Rev. D 65, 043502 (2002) arXiv:astro-ph/0107245.

[14] C. Charmousis and J-F. Dufaux, arXiv:hep-th/0311267

[15] J. F. Dufaux, J. E. Lidsey, R. Maartens and M. Sami, Phys. Rev. D, to appear, arXiv:hep-th/0404161

[16] H. Davoudiasl, J. L. Hewett, and T. G. Rizzo,
JHEP 03, 034 (2003) arXiv:hep-ph/0305086; S. L. Dubovsky and M. V. Libanov, JHEP 03, 038 (2003) arXiv:hep-th/0309131; Y. Shtanov and A. Viznyuk, arXiv:hep-th/0312261 M. N. Smolyakov, arXiv:hep-th/0403034

[17] A. Padilla, Class. Quant. Grav. 21, 2899 (2004) arXiv:hep-th/0402079.

[18] F. Cooper, A. Khare, and U. Sukhatme, Phys. Rep. 251, 267 (1995) arXiv:hep-th/9405029.

[19] A. Frolov and L. Kofman, arXiv:hep-th/0209133

[20] D. S. Gorbunov, V. A. Rubakov, and S. M. Sibiryakov, J. High Energy Phys. 10, 015 (2001) arXiv:hep-th/0108017; T. Kobayashi, H. Kudoh, and T. Tanaka, Phys. Rev. D68, 044025 (2003) arXiv:gr-qc/0305006.

[21] J. E. Lidsey, A. R. Liddle, E. W. Kolb, E. J. Copeland, T. Barreiro, and M. Abney, Rev. Mod. Phys. 69, 373 (1997).

[22] S. M. Leach and A. R. Liddle, Phys. Rev. D68, 123508 (2003) arXiv:astro-ph/0306305; S. Tsujikawa and A. R. Liddle, JCAP 03, 001 (2004) arXiv:astro-ph/0312162; S. Tsujikawa, M. Sami, and R. Maartens, arXiv:astro-ph/0406078

[23] R. Maartens, D. Wands, B. A. Bassett, and I. P. C. Heard, Phys. Rev. D62, 041301 (2000) arXiv:hep-ph/9912464.

[24] S. Tsujikawa and B. Gumjudpai, arXiv:astro-ph/0402185

[25] G. Huey and J. E. Lidsey, Phys. Lett. B514, 217 (2001) arXiv:astro-ph/0104006.

[26] G. Huey and J. E. Lidsey, Phys. Rev. D66, 043514 (2002) arXiv:astro-ph/0205236.

[27] G. F. Giudice, E. W. Kolb, J. Lesgourgues, and A. Riotto, Phys. Rev. D66, 083512 (2002) arXiv:hep-ph/0207145.

[28] D. Seery and A. N. Taylor, arXiv:astro-ph/0309152 G. Calcagni, JCAP 11, 009 (2003) arXiv:hep-ph/0310304.

[29] D. Wands, K. A. Malik, D. H. Lyth, and A. R. Liddle, Phys. Rev. D62, 043527 (2000) arXiv:astro-ph/0003278.

[30] D. H. Lyth and D. Wands, Phys. Rev. D 68, 103515 (2003) arXiv:astro-ph/0306498. 